\title{
Building a network to support people in suicide risk in Rio de Janeiro, from Primary Care to Mental Health Services
}

Rocha, Helio (hearoch@gmail.com); Minozzo, Fabiane; Teixeira, Debora; Sarmento, Laura; Fortes, Sandra

Objectives: To present The Rio de Janeiro Suicide Risk Assessment and Prevention Quick Reference Guide. To create a care flow for the suicide risk cases in the city's mental health network.

Background: It is estimated that about $90 \%$ of individuals who ended their lives committing suicide had some mental disorder, that at that time $60 \%$ of them were depressed and that $45 \%$ of people who commited suicide were attended at Primary Care (PC) in the month before their deaths. In the year before suicide, $77 \%$ from who committed suicide were attended at PC but only $32 \%$ in mental health services.

Materials and Methods: A literature review about epidemiology, assessment, prevention and treatment of suicide risk was held by a task force to organized the assignment of primary care, emergency and mental health services to approach suicide risk patients in Rio de Janeiro.
Results: The Rio de Janeiro Suicide Risk Assessment and Prevention Quick Reference Guide was developed and distributed to all units. It has been the basis to organize the assessment of suicide risk, and to develop a stepped-care approach within an integrated services network to support and treat these patients.

Conclusions: The guide is being used to train and help primary care professionals in approaching, detecting and treating patients in suicide risk, including facilitating access to specialized services.

\section{SUICIDE RISK ASSESSMENT}

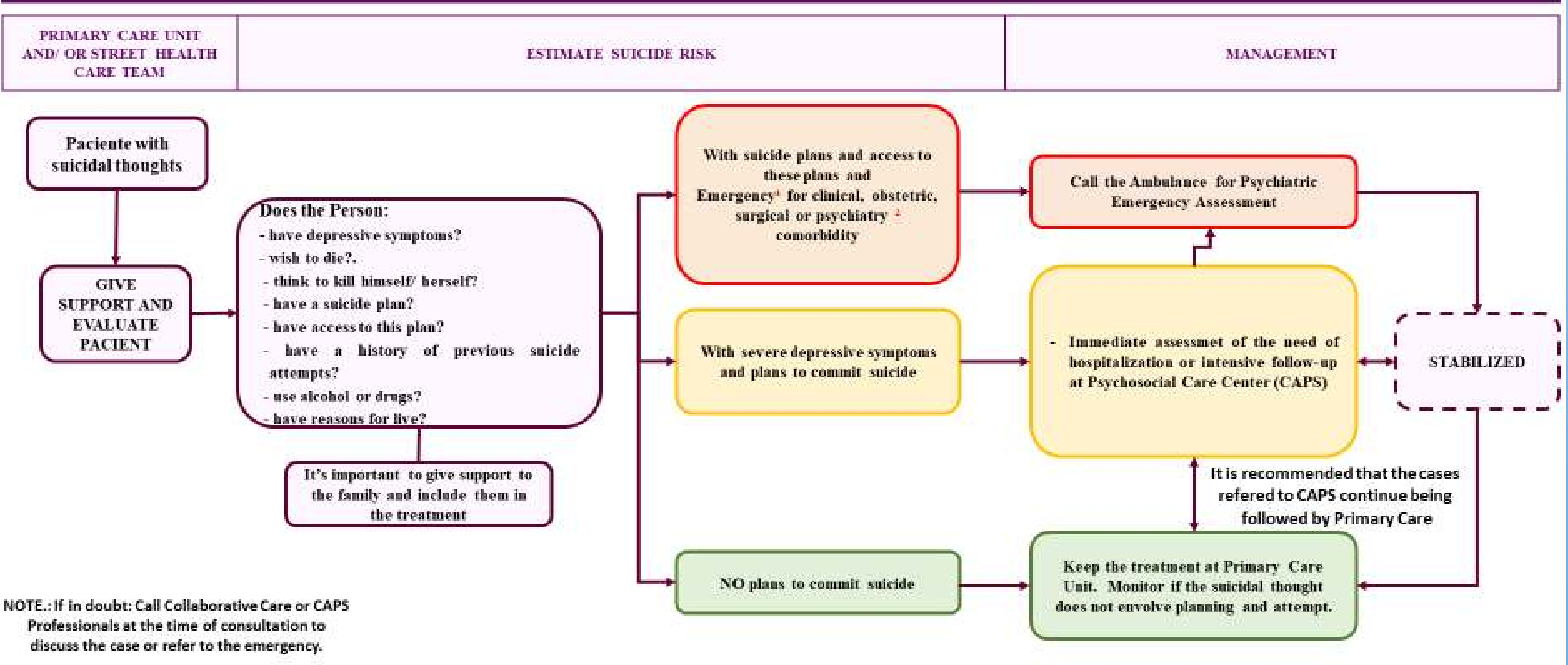

